\title{
Original
}

\section{Morphological Effects of Laser Irradiation on the Cochlea}

\author{
Gen Namba and Yasuya Nomura
}

\begin{abstract}
Argon laser irradiation was applied to the stria vascularis of the third turn through the otic capsule of guinea pigs and to the osseous spiral lamina and the organ of Corti through the round window membrane. A laser probe, $0.6 \mathrm{~mm}$ in diameter, was connected to an argon laser. A glass fiber of $200 \mu \mathrm{m}$ in diameter was encased in the probe. Each time, the power applied was $50-300 \mathrm{~mW}$ and of 0.5 -second duration. The tip of the probe was 0.5 to $1 \mathrm{~mm}$ away from the otic capsule. Twenty-six to 48 days after irradiation, the animals were killed under deep anesthesia after intravital fixation with Heidenhein-Susa solution. The conventional celloidin-embedding technique was used to make sections of $20 \mu \mathrm{m}$ thickness for microscopic observation. The spiral ligament showed atrophy and the stria vascularis disappeared in the third turn. The otic capsule remained intact. The organ of Corti was partly or completely missing. Extension of Reissner's membrane covered the atrophic spiral ligament and was connected to the basilar membrane after the spiral ligament had disappeared. Irradiation $(1 \mathrm{~W} \times 0.5$ seconds $)$ through the round window membrane destroyed the organ of Corti and fractured the bony shelf of the osseous spiral lamina with loss of dendrites of cochlear neurons. The round window membrane remained intact.
\end{abstract}

Key words: argon laser, spiral ligament, stria vascularis, strial atrophy, round window membrane

The use of lasers has changed the concepts of surgery in the field of ear, nose and throat surgery. In otology, the laser has been used successfully in stapes surgery and in other types of middle ear surgery ${ }^{1)}$. Argon lasers have been applied experimentaly to the vestibular labyrinth ${ }^{2,3)}$, and have been used clinically in the surgical treatment of intractable vertigo ${ }^{4}$. However, application of the argon laser to the cochlea has not yet been studied.

The purpose of the present investigation was to examine the effects of argon laser on the cochlea, particularly with regard to the possibility of establishment of a strial atrophy animal model and the possibility of clinical application in diseases of the ear.

\section{Materials and Methods}

Thirty guinea pigs of the Takei strain $(300 \mathrm{~g})$ were used in this series of experiments. After intraperitoneal administration of sodium pentobarbital $(40 \mathrm{mg} / \mathrm{kg})$, the cochleas of the guinea pigs were exposed by a ventral approach after a submaxillary incision and opening of the bulla.

The argon laser equipment used in these experiments was manufactured by HGM Laser

Department of Otolaryngology, Showa University School of Medicine, 1-5-8 Hatanodai, Shinagawa-ku, Tokyo 142, Japan. 
Systems (Salt Lake City, UT). A probe, $0.6 \mathrm{~mm}$ in diameter, was connected to the machine. A glass fiber, $200 \mu \mathrm{m}$ in diameter, was encased in the probe. The power range of the argon laser beam was from 50 to $300 \mathrm{~mW}$, with the duration of irradiation set at 0.5 seconds. The distance between the tip of the probe and the area to be irradiated was approximately $0.5 \mathrm{~mm}$.

Two areas in the cochlea were irradiated by the argon laser: 1) the lateral wall of the cochlea and 2) the inferior of the cochlea through the round window membrane. To irradiate the lateral wall, the tip of the probe was directed to the dark band due to pigment in the stria vascularis.

The animals were either killed under deep anesthesia immediately after irradiation or kept alive for 26 to 48 days. The animals were intravitally fixed with Heidenhein-Susa solution. After being fixed and washed with tap water, the temporal bone was removed and transferred to $5 \%$ trichloroacetic acid solution for decalcification. Decalcified specimens were neutralized with $5 \%$ sodium sulfate solution and then washed thoroughly. The conventional celloidin method was used to section specimens at a thickness of $20 \mu \mathrm{m}$. Sections were stained with hematoxylin and eosin (H.E.) before mounting in Malinol (Muto Pure Chemicals Co. Ltd., Tokyo).

Some of the temporal bones in the round window membrane irradiation group were dissected to remove the round window membrane and the osseous spiral lamina with the basilar membrane. In this group, the power of the argon laser beam was as high as $1.0 \mathrm{~W}$. These specimens were observed with a surface preparation technique after the cochlea was dissected. After dehydration, small pieces of dissected specimens were mounted on a slide glass with Malinol.

\section{Results}

\section{Irradiation of the otic capsule}

The lateral wall of the membranous cochlea consists of the spiral ligament and stria vascularis (Fig. 1). The otic capsule covers these structures. The purpose of application of an argon laser to the bony wall is to produce localized atrophy of the stria vascularis. The tip of the laser probe was advanced toward the otic capsule of the third turn of the cochlea, approximately $0.5 \mathrm{~mm}$ away from the bony surface. The target was the stria vascularis. The power applied was 50 to $300 \mathrm{~mW}$ and the duration was 0.5 seconds.

The otic capsule did not show any changes immediately after irradiation, the power density of which was $0.617 \mathrm{~W} / \mathrm{mm}^{2}(50 \mathrm{~mW} \times 0.5 \mathrm{sec})$. However, the otic capsule was superficially damaged at the area of impact and turned black when irradiated with the same power density after the surface was stained with $2 \%$ mercurochrome solution. Absorption of the argon laser was enhanced at the impact area by red coloring on the surface of the otic capsule.

Celloidin-embedded specimens of the irradiated area showed an acellular, atrophic spiral ligament. The tissue was partially disrupted. The stria vascularis had disappeared. The atrophic spiral ligament was covered by a single cell layer, which was a continuation of the normal Reissner's membrane. The cell layer covering the spiral ligament had not ruptured. Thus, the integrity of the endolymphatic compartment was maintained (Fig. 2).

The organ of Corti showed partial loss of hair cells. When present, the outer hair cells were devoid of nuclei (Fig. 3). Hensen's cells were not influenced by a beam of 50-120 mW. 


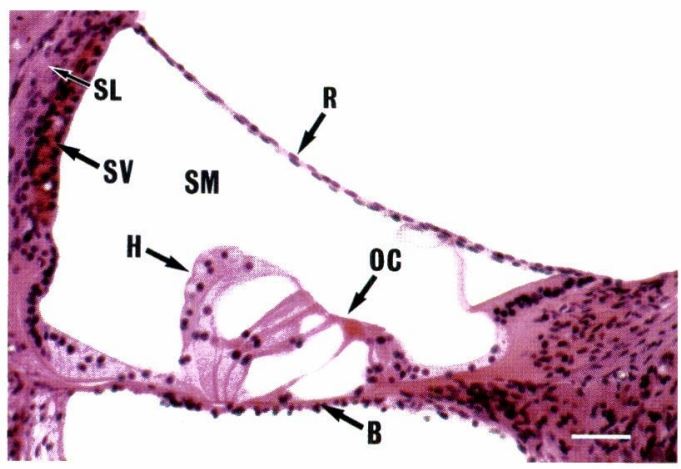

Fig. 1. The third turn of the non-irradiated cochlea (Control). Scale: $50 \mu \mathrm{m} . \times 64$. SV: stria vascularis, SL: spiral ligament, R: Reissner's membrane, OC: organ of Corti, H: Hensen's cells, SM: scala media, B: basilar membrane.

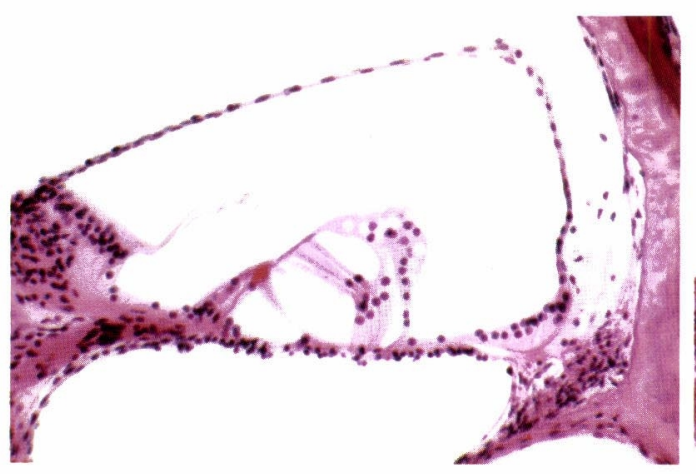

Fig. 2.

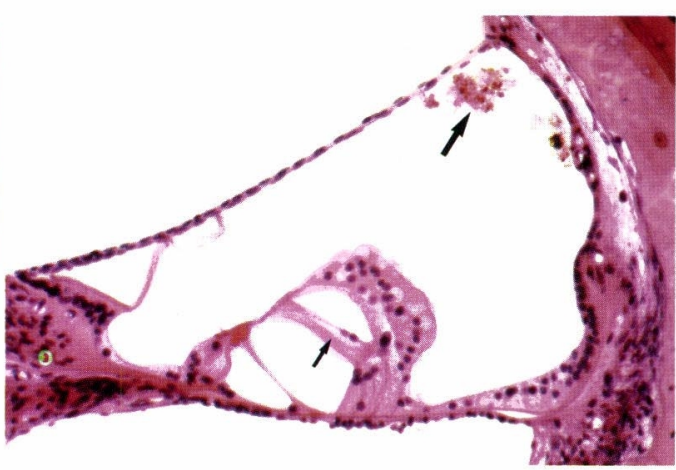

Fig. 3.

Fig. 2. Loss of stria vascularis with partial loss of spiral ligament. The first row of hair cells is missing. Thirty-five days after irradiation to the otic capsule $(90 \mathrm{~mW} \times 0.5 \mathrm{sec})$. Third turn. $\times 64$.

Fig. 3. Free floating, disintegrated strial tissue in scala media (large arrow). Although stereocilia are present, there is fusion of cell bodies without nuclei (small arrow). Thirty days after irradiation $(50 \mathrm{~mW} \times 0.5 \mathrm{sec})$. Third turn. $\times 64$.

However, some Hensen's cells were lost when more power was applied. The inner hair cells were usually spared. The spiral ganglion cells were normal. The dendrites of cochlear neurons disappeared when the organ of Corti was missing.

The otic capsule of the third turn was $102 \mu \mathrm{m}$ in thickness (average of 12 animals) and did not show morphologic changes. Apparently, the laser beam passed through the bony wall, producing atrophy of the spiral ligament and disappearance of the stria vascularis. In some animals, there were fragments of disintegrated stria vascularis in the scala media. The stria vascularis of these specimens was either partially or completely missing (Figs. 3, 4).

When more heat was applied to the otic capsule, a charred area with a lucent area adjacent to it developed. Even though such a burned area was produced by irradiation, the periosteal layer remained intact (Fig. 5A, B). In this specimen, the spiral ligament was 


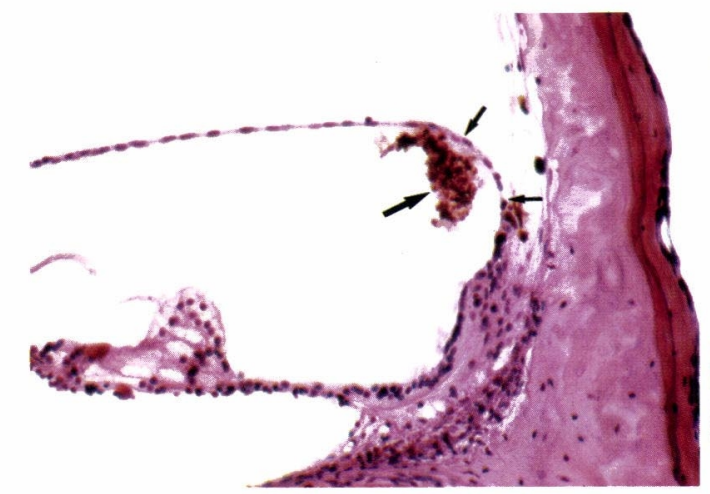

Fig. 4.

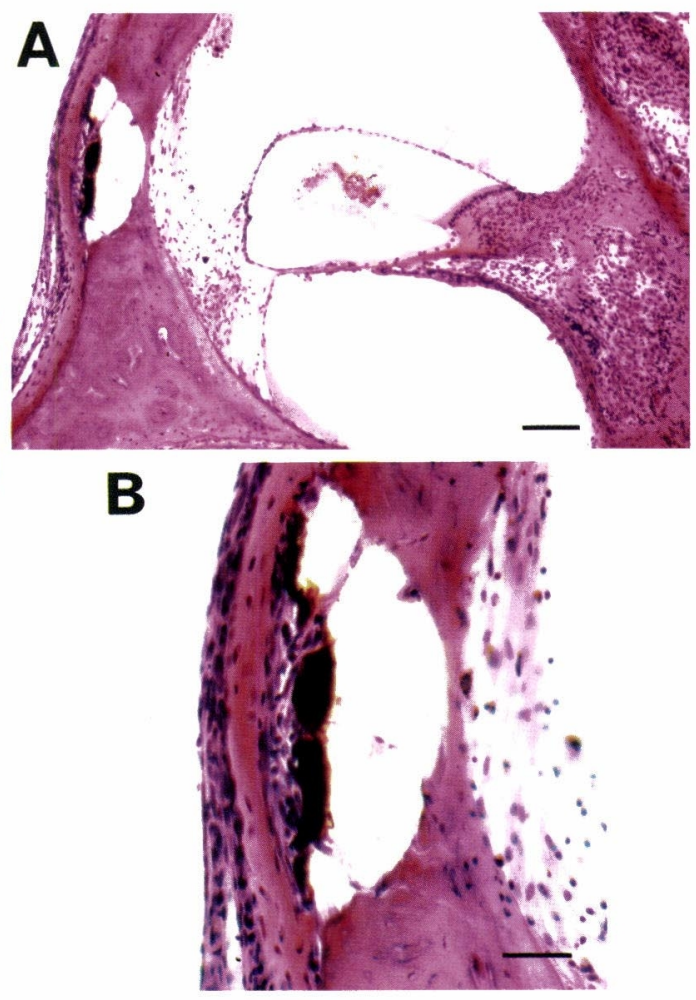

Fig. 5.

Fig. 4. Detached stria vascularis in scala media (large arrow). Reissner's membrane shows extension to cover the defective spiral ligament (small arrows). Forty-eight days after irradiation $(120 \mathrm{~mW} \times 0.5 \mathrm{sec})$. Third turn. $\times 64$.

Fig. 5. A: Changes in the otic capsule and inside of cochlea. A charred area is present beneath the normal periosteum. There is a wide lucent area adjacent to the charred area. There is loss of dendrites in the osseous spiral lamina. Fifteen days after irradiation $(300 \mathrm{~mW} \times 0.5 \mathrm{sec})$. The second turn was irradiated in this animal. Scale: $100 \mu \mathrm{m} . \times 26$.

B: High-power view of A. Charred and lucent areas in the enchondral layer. The periosteal layer remains intact. Maximum diameter of the lucent area is $270 \mu \mathrm{m}$. Scale: $50 \mu \mathrm{m} . \times 64$.

partly atrophic and partly defective. The stria vascularis and the organ of Corti were completely missing. The osseous spiral lamina appeared empty, indicating almost complete loss of dendrites of cochlear neurons. The contour of the endolymphatic compartment was preserved.

When damage was more severe, resulting in disappearance of the spiral prominence and basilar crest, Reissner's membrane seemed to maintain the integrity of the endolymphatic compartment by connecting it to the basilar membrane (Fig. 6).

\section{Irradiation through the round window membrane}

The tip of the laser probe was advanced toward the round window membrane. The distance between the tip and the membrane was about $0.5 \mathrm{~mm}$. The osseous spiral lamina and the organ of Corti were the targets. 


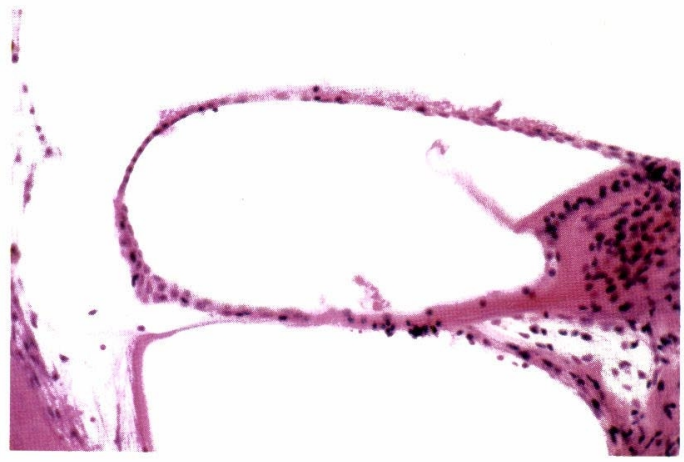

Fig. 6. Marked loss of spiral ligament. Twenty-six days after irradiation $(300 \mathrm{~mW} \times 0.5 \mathrm{sec})$. Third turn. $\times 64$.
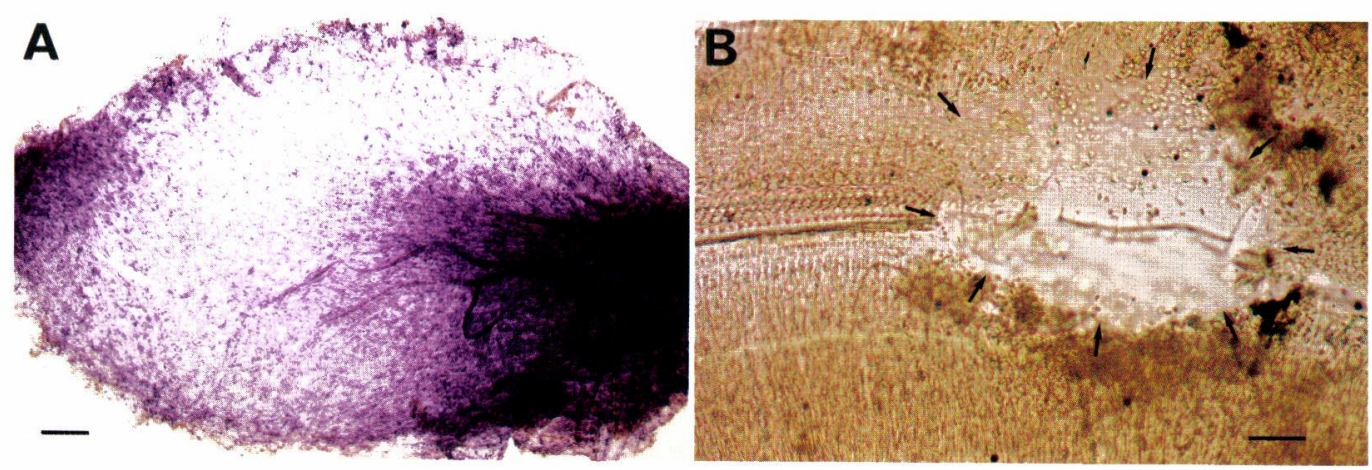

Fig. 7. Laser irradiation of the organ of Corti through the round window membrane.

A: The intact round window membrane. Argon laser irradiation $(1.0 \mathrm{~W} \times 0.5 \mathrm{sec})$ was applied to the cochlea through the membrane. The small cleft is an artifact produced during specimen processing. H.E. stain. Scale: $100 \mu \mathrm{m} . \times 26$.

B: The organ of Corti is damaged (arrows). The specimen was removed immediately after irradiation. Scale: $50 \mu \mathrm{m} . \times 200$.

The round window membrane did not show either a burned spot or a perforation by the argon laser beam, the energy of which was $6.15 \mathrm{~J} / \mathrm{mm}^{2}(1.0 \mathrm{~W} \times 0.5 \mathrm{sec})$ (Fig. 7A). Changes were observed in the osseous spiral lamina, basilar membrane, organ of Corti, stria vascularis, and Reissner's membrane, depending on which areas were irradiated (Fig. 7B).

When the osseous spiral lamina was irradiated, its bony shelves were fractured and the dendrites disappeared (Figs. 8A, B). The organ of Corti was completely lost or in the process of degeneration. The argon laser was applied 5 times $(1 \mathrm{~W} \times 0.5 \mathrm{sec}$, each time) through the round window membrane. Cyst formation of the stria vascularis was not due to a direct effect of laser irradiation, but a secondary effect of irradiation to the neighboring tissues, as the stria vascularis could not be irradiated directly through the round window because of its anatomic situation. 

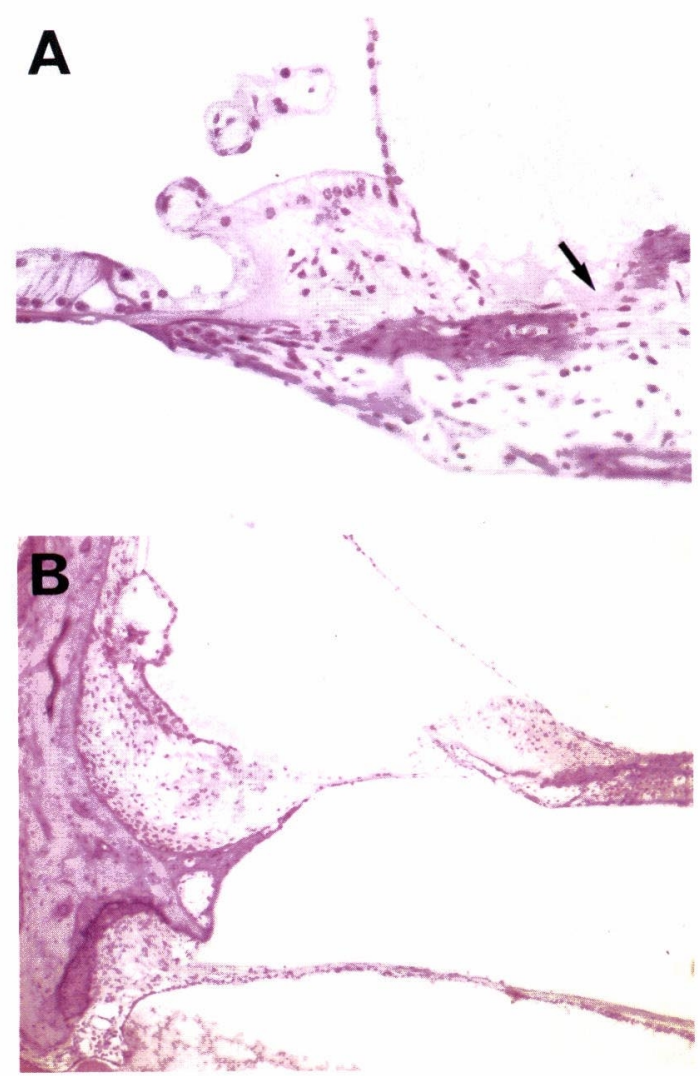

Fig. 8. Laser irradiation of the osseous spiral lamina through the round window membrane. A: There is loss of dendrites of cochlear neurons. Fracture of the upper bony shelf is possibly due to microexplosion within the osseous spiral lamina (arrow). The limbus and tectorial membrane show degeneration. $\times 64$.

$\mathrm{B}$ : There is a fracture of the osseous spiral lamina, loss of dendrites, loss of the organ of Corti, and cyst formation in the stria vascularis.

The round window membrane is intact. Two days after irradiation through the round window $(1 \mathrm{~W} \times 0.5 \mathrm{sec}, 5$ times $) . \times 26$.

\section{Discussion}

The wavelength of the argon laser is between 457.9 and $514.5 \mathrm{~nm}$. This is in the bluegreen color range of the visible light spectrum. The argon laser beam passes through water and inner ear fluids without the optic energy converting to heat. The beam penetrates water for a distance of about $10^{4} \mathrm{~mm}$ in clear water before diminishing to $1 / 10$ of its original intensity. The diameter of the tip of the probe used in the present study was $200 \mu \mathrm{m}$. When a material is irradiated $0.5 \mathrm{~mm}$ away from the tip by a beam of $1.0 \mathrm{~W}$, the spot size is $320 \mu \mathrm{m}$ and power density is $12.3 \mathrm{~W} / \mathrm{mm}^{2}$.

The present study demonstrates that an argon laser beam of 50 to $90 \mathrm{~mW}$ and 0.5 -second duration passes through the otic capsule and causes morphologic changes in the spiral ligament and stria vascularis, when the bony wall is thin.

Three mechanisms by which argon laser irradiation causes such tissue changes may be 
considered. One possible mechanism is heat generated in the otic capsule. Atrophy of the spiral ligament and the stria vascularis is due to heat produced in the otic capsule. This is likely when the otic capsule has a burned spot. However, if there is no burned area, heat cannot be considered a cause.

A second possible mechanism for these tissue changes is local circulation disturbance. Because argon laser irradiation is strongly absorbed by hemoglobin, coagulation occurs in small arterioles and capillaries in the spiral ligament and stria vascularis, with ensuing degeneration of the tissues. Alkaline phosphatase activity is present in the wall of the strial capillaries. However, it becomes inactive immediately after irradition"). All vessels in the impact area are later destroyed ${ }^{6}$.

A third possible mechanism by which argon laser irradiation causes tissue changes is tissue specificity. The argon laser beam may have a specific photodynamic effect on the stria vascularis or spiral ligament or both. The argon laser is known to have tissue specificity. It was previously demonstrated that the macula sacculi disappeared after argon laser irradiation through the saccular wall, which remained intact ${ }^{5)}$. The macula utriculi also disappeared when irradiated from below through the stapedectomized oval window ${ }^{2}$. In the maculae sacculi and utriculi, initial changes were observed in the sensory epithelium that was elevated from the supporting cells. Possibly, the energy was absorbed between the sensory epithelium and the supporting cells, resulting in steam formation followed by elevation of the sensory epithelium. The maculae sacculi and utriculi completely disappeared over several weeks, while the contour of the membranous vestibular labyrinth remained intact.

Stahle, Högberg, and Engström have suggested that the laser beam might be transmitted through the otic capsule, leaving it undisturbed, but producing changes in the stria vascularis beneath the impact point ${ }^{i}$. The present study revealed that the argon laser beam passed through the periosteal layer of the otic capsule, the same way it passed through the round window membrane. When the cortical bone was thin, the beam penetrated to the spiral ligament. However, when the otic capsule was thick, as seen in the second and basal turns, the beam could not reach the spiral ligament without burning the otic capsule, but the periosteal layer remained intact.

There were floating segments of disintegrated stria vascularis in the scala media. Coverage of the atrophic spiral ligament by a single cell layer was complete. This indicates that Reissner's membrane starts to cover the atrophied spiral ligament quickly before the stria vascularis has completely disappeared. Atrophy of the spiral ligament and disappearance of the stria vascularis occurred within a month after laser irradiation. It was also observed that Reissner's membrane was connected to the basilar membrane when the spiral ligament was largely lost. An insult to these tissues initiates growth of Reissner's membrane.

Using a photochemical reaction, Hoshino et al. ${ }^{8}$ produced a small localized lesion in the stria vascularis of the guinea pig. They illuminated the otic capsule using a green light beam ( $1 \mathrm{~mm}$ in diameter) supplied by a xenon lamp for 10 minutes, after infusion of rose bengal $(20 \mathrm{mg} / \mathrm{kg})$ intravenously. They reported that the hair cells were preserved 3 hours after illumination. However, the hair cells were found to have degenerated 1 week after illumination.

In the present study, we found that the outer hair cells in the organ of Corti are vulnerable. The stereocilia on the outer hair cell remained at the light microscopic level, even though the cell bodies showed degeneration. 
Strial atrophy is one of the pathologic changes observed in the human temporal bone ${ }^{9)}$. It produces a type of presbycusis, presenting clinically as a flat sensorineural hearing loss in audiograms. In a study of human temporal bones, the spiral ligament was usually preserved in the cochlea of patients with strial atrophy. The present study in guinea pigs indicates that severe atrophy or loss of the spiral ligament occurs together with severe atrophy or disappearance of the stria vascularis. These findings differ from those observed in human temporal bones.

The present study, however, suggests that strial atrophy may be produced when argon laser is directed to the otic capsule with lower power density. This remains to be confirmed.

Microscopic examination after application of argon laser through the round window revealed that the dendrites of cochlear neurons in the osseous spiral lamina disappeared after irradiation, whereas the round window membrane itself remained intact.

In the future, clinical application of this method will be useful for the treatment of Meniere's disease as an alternative to endocochlear shunt surgery and for the treatment of patients with tinnitus in whom cochlear neurectomy is indicated.

\section{References}

1) Nissen AJ: Laser applications in otologic surgery. ENT J, 74: 477-482 (1955)

2) Okuno T, Nomura $Y$ and Young YH: Ablation of otolithic organs with argon laser. Acta Otolaryngol (Stockh), Suppl 481: 607-609 (1971)

3) Oki S, Nomura $Y$, Sugio $Y$ and Young $Y H$ : Occlusion of the semicircular canal by argon laser. J Clin Laser Med Surg, 14: 393-398 (1996)

4) Nomura $\mathrm{Y}$, Okuno $\mathrm{T}$ and Mizuno M: Treatment of vertigo using laser labyrinthectomy. Acta Otolaryngol (Stockh), 113: 261-262 (1993)

5) Nomura Y, Hara M and Okuno T: Application of argon laser to the inner ear. Acta Otolaryngol (Stockh), 105: 439-444 (1988)

6) Sugar JO, Stahle J and Högberg L: Laser irradiation of the stria vascularis. Arch Otolaryngol, 99: $330-336$ (1974)

7) Stahle J, Högberg L and Engström B: The laser as a tool in inner-ear surgery. Acta Otolaryngol (Stockh), 73: 27-37 (1972)

8) Hoshino T, Iwasaki S and Wo R: Focal cochlear damage induced by photochemical reaction. I. Damage and cochlear function. Annual Report of Research Committee of Acute Profound Deafness 1995, 25-26 (1996)

9) Schuknecht HF: Pathology of the Ear. Second Edition, Lea \& Febiger, Philadelphia, p 419 (1993)

[Received January 9, 1997: Accepted January 31, 1997] 